\title{
Ursolic Acid Suppresses Cholesterol Biosynthesis and Exerts Anti-Cancer Effects in Hepatocellular Carcinoma Cells
}

\author{
Geon-Hee Kim ${ }^{1,2}$, Sang-Yeon Kan ${ }^{1,2}$, Hyeji Kang ${ }^{1,2}$, Sujin Lee ${ }^{1,2}$, Hyun Myung Ko ${ }^{3}{ }^{\circledR}$, \\ Ji Hyung Kim ${ }^{4}$ and Ji-Hong Lim ${ }^{1,2, *}$ \\ 1 Department of Applied Life Science, Graduate School of Konkuk University, College of Biomedical \& Health \\ Science, Konkuk University, Chungju 27478, Korea; rlarjsgml4@kku.ac.kr (G.-H.K.); \\ hsb6477@kku.ac.kr (S.-Y.K.); kkang@kku.ac.kr (H.K.); 201341532@kku.ac.kr (S.L.) \\ 2 Diabetes and Bio-Research Center, Konkuk University, Chungju 27478, Korea \\ 3 Department of Life Science, College of Science and Technology, Woosuk University, 66 Daehak-ro, \\ Jincheon-eup, Chungcheongbuk-do 27841, Korea; greatmen00@hanmail.net \\ 4 College of Life Sciences and Biotechnology, Korea University, Seoul 02841, Korea; jay_kim@korea.ac.kr \\ * Correspondence: jhlim@kku.ac.kr; Tel.: +82-43-840-3567; Fax: +82-43-840-3929
}

Received: 5 August 2019; Accepted: 25 September 2019; Published: 26 September 2019

\begin{abstract}
Abnormally upregulated cholesterol and lipid metabolism, observed commonly in multiple cancer types, contributes to cancer development and progression through the activation of oncogenic growth signaling pathways. Although accumulating evidence has shown the preventive and therapeutic benefits of cholesterol-lowering drugs for cancer management, the development of cholesterol-lowering drugs is needed for treatment of cancer as well as metabolism-related chronic diseases. Ursolic acid (UA), a natural pentacyclic terpenoid, suppresses cancer growth and metastasis, but the precise underlying molecular mechanism for its anti-cancer effects is poorly understood. Here, using sterol regulatory element (SRE)-luciferase assay-based screening on a library of 502 natural compounds, this study found that UA activates sterol regulatory element-binding protein 2 (SREBP2). The expression of cholesterol biosynthesis-related genes and enzymes increased in UA-treated hepatocellular carcinoma (HCC) cells. The UA increased cell cycle arrest and apoptotic death in HCC cells and reduced the activation of oncogenic growth signaling factors, all of which was significantly reversed by cholesterol supplementation. As cholesterol supplementation successfully reversed UA-induced attenuation of growth in HCC cells, it indicated that UA suppresses HCC cells growth through its cholesterol-lowering effect. Overall, these results suggested that UA is a promising cholesterol-lowering nutraceutical for the prevention and treatment of patients with HCC and cholesterol-related chronic diseases.
\end{abstract}

Keywords: Ursolic acid; SREBP2; Cholesterol; Hepatocellular carcinoma

\section{Introduction}

Hepatocellular carcinoma (HCC) is the most common type of primary liver cancer, which is closely associated with chronic liver diseases, particularly viral hepatitis and metabolic steatohepatitis, and is the third most common cause of cancer-related deaths worldwide [1-3].

Ursolic acid (UA) is a pentacyclic terpenoid and a secondary plant metabolite, usually found in the holy basil (Ocimum sanctum L.), thyme (Thymus vulgaris L.), lavender (Lavandula augustifolia), catnip (Nepeta sibthorpii), peppermint leaves (Mentha piperita L.), or fruit peel [4,5]. It has various benefits for the prevention and treatment of chronic human diseases, such as diabetes, cardiovascular, arthritis, atherosclerosis, obesity, and cancer [5]. 
UA is known to induce cell cycle arrest and apoptosis, suppress angiogenesis and metastasis, and diminish chemoresistance in several cancers, including lung cancer [6,7], breast cancer [8,9], prostate cancer [10], colon cancer [11,12], liver cancer [13,14], gastric cancer [15], and melanoma [16,17]. In addition, the anti-cancer effects of UA have been observed in animal models, such as subcutaneous xenograft (HCT116 and A549), orthotopic xenograft (HCT116 and Panc-28), transgenic adenocarcinoma of mouse prostate (TRAMP), and DMBA-induced skin cancer [12,17-19].

The evidences from previous studies suggest that UA exerts its anti-cancer effects through the suppression of oncogenic growth signaling, such as that via phosphoinositide 3-kinase (PI3K)/protein kinase B (AKT) and epidermal growth factor receptor (EGFR)/mitogen-activated protein kinase (MAPK) pathways, and oncogenic transcription factors, such as nuclear factor kappa-light-chain-enhancer of activated B cells (NF-kB), signal transducer and activator of transcription 3 (STAT3), and hypoxia-inducible factor- $1 \alpha$ (HIF-1 $\alpha$ ), in several types of cancer [5]. However, the precise molecular mechanism by which UA affects these cancer-promoting signaling molecules and transcription factors is poorly understood.

Mammalian cells synthesize cholesterol through a series of 21 enzymatic steps, including the mevalonate (MVA) pathway, generating various metabolites that are required for maintenance of physiological and developmental processes [20]. Enriched cholesterol is commonly observed in lipid raft microdomains of the cell membrane, which is involved in various cellular functions, such as the regulation of cell adhesion, migration, and growth signaling, e.g., PI3K/AKT and EGFR/MAPK [21-23]. Therefore, the increase in intracellular cholesterol level due to dysregulation of its biosynthetic pathways is a common feature of cancer, and the evidence suggests that cholesterol is a critical component in the progression of various cancers, including breast, prostate, liver, and colorectal cancer [21,24].

Although the main source of cholesterol is diet, intracellular cholesterol levels are carefully regulated and balanced by sterol regulatory element-binding protein 2 (SREBP2)-mediated transcriptional programming [25]. When intracellular cholesterol levels are sufficient, SREBP2 is not processed to its maturation, and cholesterol synthesis is not stimulated. Conversely, when the cells sense low cholesterol levels, SREBP2 maturation is induced, followed by its translocation into the nucleus for activation of its target genes, including those encoding hydroxymethylglutaryl (HMG)-CoA synthase 1 (HMGCS1), HMG-CoA reductase (HMGCR), farnesyl diphosphate synthase (FDPS), and mevalonate diphosphate decarboxylase (MVD), for de novo cholesterol synthesis [25].

Statins, inhibitors of HMG-CoA reductase, which is the rate-limiting enzyme in cholesterol biosynthesis, are widely used as cholesterol-lowering drugs [26,27]. Emerging evidence from tissue culture, animal, and clinical studies indicates that several statins, such as strovastatin, fluvastatin, and simvastatin, stimulate cell cycle arrest, apoptotic cell death, and the suppression of EMT and cancer stemness in several types of proliferating cancer cells, including hepatocellular carcinoma, breast, prostate, glioma, ovarian, and colorectal cancer cells [28-31]. Recent meta-analyses have revealed that statins have a beneficial effect with respect to reduced cancer-related mortality on multiple cancer types, including hepatocellular carcinoma (HCC), breast, lung, prostate, colorectal, and kidney cancer [32-39]. Manthravadi et al. reported that statin use is associated with improved recurrence-free survival (RFS), cancer-specific survival, and overall survival in breast cancer patients [33]. A meta-analysis in patients with HCC in a U.S. population revealed that ever-use of statins has a significant inverse association with HCC development [34]. In addition, a reduced cancer-related mortality among statin users, in comparison with those who had never used statins, was observed for 13 cancer types [40]. Consistent with these observations, accumulated data from a meta-analysis revealed that statins may have beneficial effects for the prevention and treatment of multiple types of cancer.

A growing body of preclinical, epidemiological, and clinical evidence has shown the various side effects of the most commonly used cholesterol-lowering nutraceuticals [41]. However, efforts to develop cholesterol-lowering nutraceuticals, which exert anti-cancer effects without side effects, are needed for effective cancer prevention and treatment. The present study identified UA as a cholesterol-lowering drug using a library of 502 natural compounds. In addition, transcriptional activation of SREBP2 and 
its cholesterol biosynthesis-related target genes in UA-treated HCC cells was observed. Consistent with this cholesterol-lowering effect of UA, this study also found that UA had a negative effect on the oncogenic AKT and MAPK signaling pathway and cell viability, thereby increasing the incidence of cell cycle arrest and apoptotic death in HCC cells. Overall, our results suggest that UA may be a promising cholesterol-lowering drug to achieve prevention and treatment in patients with HCC.

\section{Results and Discussion}

\subsection{UA Identified as A SREBP2 Activator}

Sterol regulatory element-binding protein 2 (SREBP2) undergoes maturation and activation upon cholesterol depletion, subsequently triggering the enhancement of intracellular cholesterol biosynthesis, for maintaining cholesterol homeostasis [25]. Paradoxically, statins, cholesterol-lowering drugs, activate SREBP2 and its target genes related to cholesterol biosynthesis, such as HMGCS1, HMGCR, MVD, and FDPS, in order to increase intracellular cholesterol levels [42,43]. Thus, screening for a transcriptional activator of SREBP2 is a promising way to identify novel cholesterol-lowering small molecules. Here, this study attempted to identify a potential SREBP2 transcriptional activator using a SREBP2-binding sequence containing a sterol-responsive element (SRE), by conducting a luciferase assay on a library of 502 natural compounds. This study identified 24 compounds that increased the SRE luciferase activity to levels higher than that of the vehicle, which served as a negative control (Figure 1A). An additional luciferase assay to validate the larger screening also showed that these 24 compounds activated SRE-luciferase activities (Figure 1B). In this screening, UA, daidzin, and prostaglandins were found to highly activate the SRE-luciferase activity. In addition, a dose-dependent increase in SRE-luciferase activity was also observed in UA-treated SK-HEP-1, hepatocellular carcinoma (HCC) cells (Figure 1C), suggesting that UA is a potential cholesterol-lowering and SREBP2-activating natural compound. Consistent with our finding, the evidence from multiple sources suggests that UA, as a pentacyclic terpenoid, exhibits various beneficial pharmacological effects against metabolism-related chronic diseases, such as obesity [44], liver failure [45], and skeletal muscle atrophy [46]. Indeed, previous reports have shown that UA significantly decreases the blood cholesterol and triglyceride levels in a high-fat-diet-induced obesity animal model $[47,48]$. UA has also been found to decrease the liver triglyceride and free fatty acid levels in hepatic steatosis and liver fibrosis models $[49,50]$. Thus, the identification of UA as a SREBP2 activating small molecule could provide useful insights into the precise molecular mechanism underlying the beneficial effects of UA in various chronic human diseases. 
A

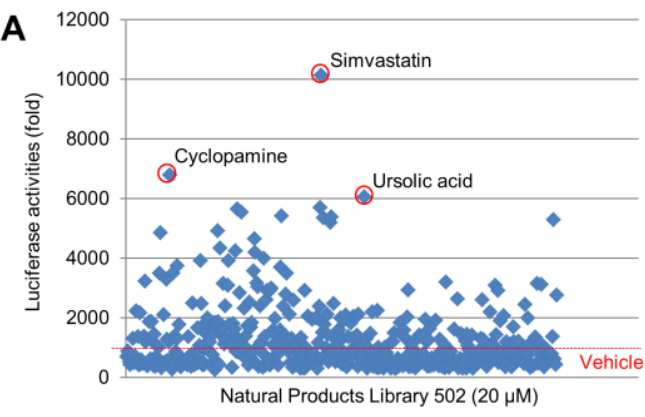

C

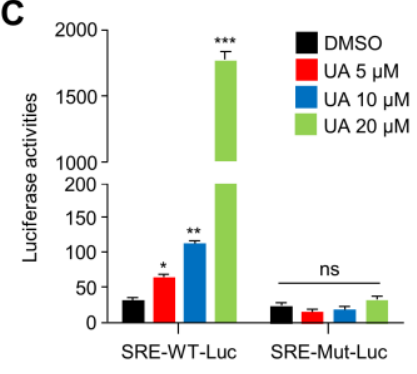

B

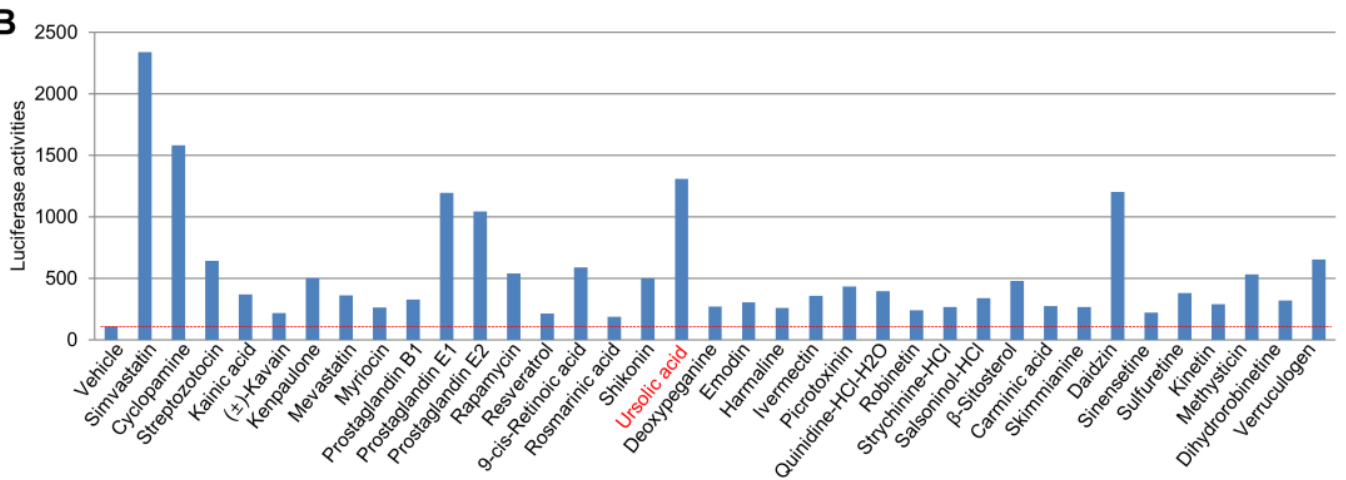

Figure 1. Luciferase assay-based screening for natural compounds that activate the sterol regulatory element-binding protein 2 (SREBP2). (A) Wild-type sterol regulatory element (SRE)-luciferase vector was transiently expressed in HEK293T cells, and incubated for $24 \mathrm{~h}$ with dimethyl sulfoxide (DMSO), as a negative control, or one of the 502 natural compounds. Each compound was dissolved in dimethyl sulfoxide (DMSO) and used at a final concentration of $20 \mu \mathrm{M}$. The values represent the mean from the two independent experiments performed. (B) HEK293T cells expressing SRE-luciferase vector were incubated for $24 \mathrm{~h}$ with 25 of the 502 library compounds, selected during the first screening. Simvastatin $(20 \mu \mathrm{M})$ was used as a positive control and DMSO (vehicle) was used as a negative control. The values represent the mean from two independent experiments performed in triplicate. (C) SK-HEP-1 cells expressing SRE-WT (wild type of SRE) or SRE-MT (mutant of SRE)-luciferase vector were incubated for $24 \mathrm{~h}$ with ursolic acid (UA) at different concentrations, as indicated. The values represent the mean $\pm \mathrm{SD}$ of three independent experiments performed in triplicate; ${ }^{*} p<0.05,{ }^{* *} p<0.01$, and ${ }^{* * *} p<0.001$.

\subsection{UA Induces the Expression of Cholesterol Biosynthesis-Related Genes and Enzymes}

To investigate whether UA activates SREBP2, the expression of cholesterol biosynthesis-related genes was analyzed in the absence or presence of UA. The increased expression was observed for genes related to cholesterol biosynthesis (HMGCS1, HMGCR, MVD, FDPS, and SREBP2), fatty acid synthesis (SREBP1a and SREBP1c), and cholesterol uptake (LDL-R) in UA-treated SK-HEP-1 cells, and this UA-induced gene expression was similar to that observed with simvastatin treatment (Figure 2A). Given that increased expression of SREBP2 and cholesterol biosynthesis-related genes is a compensatory mechanism induced by cholesterol-lowering drugs [51,52], our results indicate that UA also had cholesterol-lowering effects similar to statins. Similarly, the increased expression of proteins such as FDPS, HMGCR, HMGCS1, and SREBP2 was observed in UA-treated SK-HEP1 and Hep3B cells (Figure 2B). UA was also found to increase the expression of cholesterol biosynthesis-related genes in Huh7 and Hep3B cells (Figure 2C), suggesting that the activation of SREBP2 and the expression of its target genes upon UA treatment may be a general effect, at least in HCC cells. Therefore, the next crucial question is whether the observed increase in the expression of cholesterol biosynthesis-related genes is indeed caused by the cholesterol-lowering effect of UA. To address this question, the expression of cholesterol biosynthesis-related genes upregulated by UA treatment was analyzed in the absence or presence of water-soluble cholesterol supplementation. Interestingly, the supplementation of water-soluble cholesterol was found to diminish the upregulation of cholesterol biosynthesis-related 
genes by UA treatment (Figure 2D). In addition, this study examined whether UA can act like statins to decrease intracellular cholesterol level. Interestingly, the decreased intracellular cholesterol levels were observed in $5 \mu \mathrm{M}$ of UA-treated SK-HEP-1, Huh7 and Hep3B cells (Figure 2E). These results indicate that UA increases the expression of cholesterol biosynthesis-related genes, and this may be caused by its cholesterol-lowering effect in HCC cells.
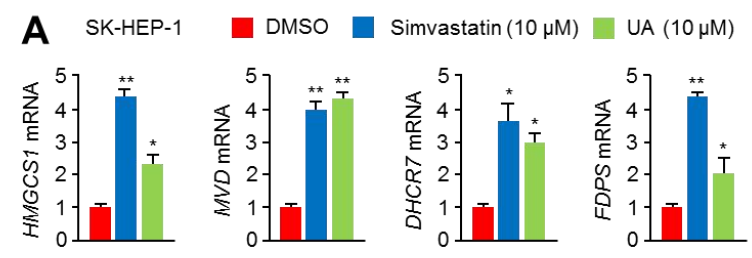

B
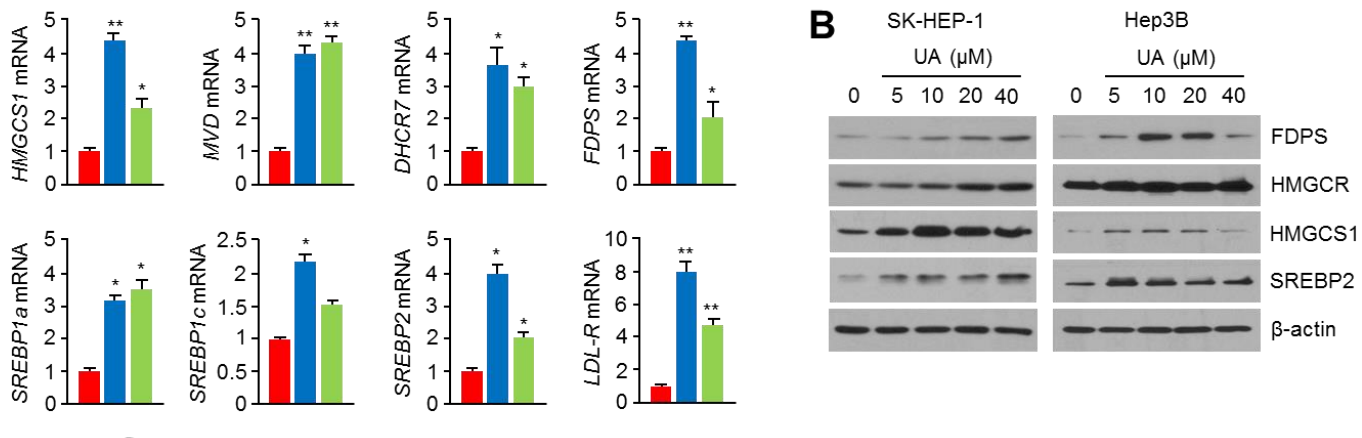

C Huh7
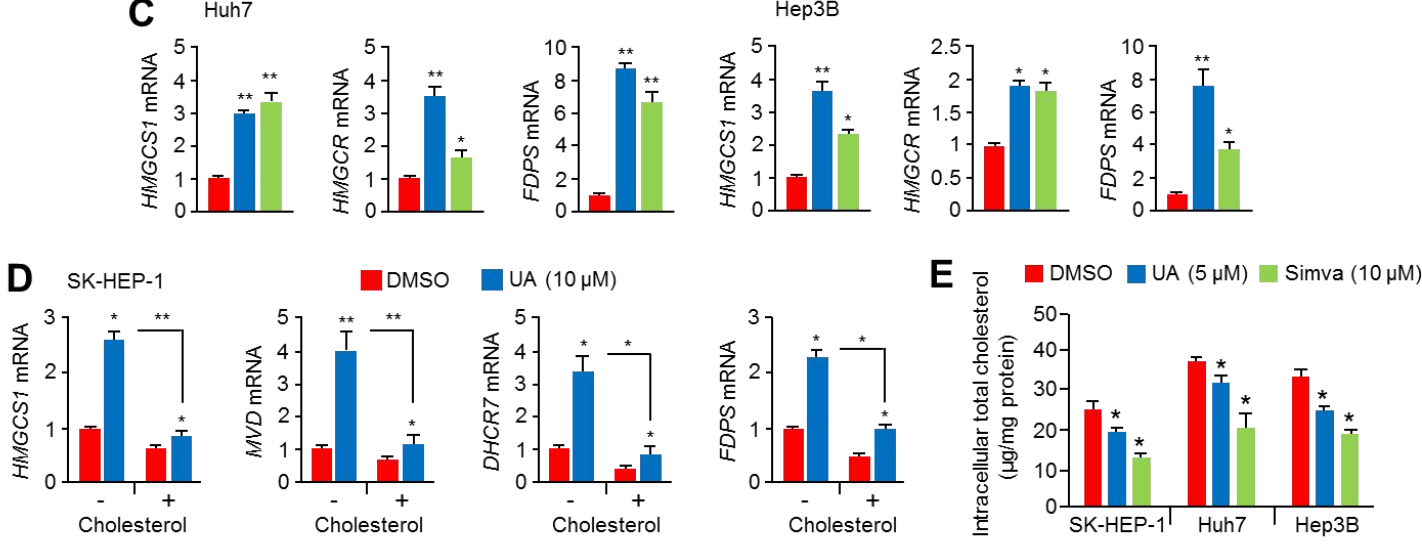

Figure 2. UA increases the expression of cholesterol biosynthesis-related genes and enzymes in hepatocellular carcinoma (HCC) cells. (A) SK-HEP-1 cells were incubated with UA $(20 \mu \mathrm{M})$ or simvastatin $(10 \mu \mathrm{M})$ for $24 \mathrm{~h}$. The mRNA levels were measured by quantitative real-time polymerase chain reaction (PCR). The values represent the mean \pm SD from three independent experiments performed in duplicate; ${ }^{*} p<0.05$ and ${ }^{* *} p<0.01$. (B) SK-HEP-1 and Hep3B cells were incubated with $\mathrm{UA}$ for $24 \mathrm{~h}$ at different concentrations, as indicated. Protein expression was measured by western blotting. (C) Huh7 and Hep3B cells were incubated with UA $(20 \mu \mathrm{M})$ or simvastatin $(10 \mu \mathrm{M})$ for $24 \mathrm{~h}$, following which, the mRNA levels were measured. The values represent the mean $\pm \mathrm{SD}$ from three independent experiments performed in duplicate; ${ }^{*} p<0.05$ and ${ }^{* *} p<0.01$. (D) SK-HEP-1 cells were incubated with or without $0.5 \mathrm{mM}$ of water-soluble cholesterol for $6 \mathrm{~h}$ prior to UA $(20 \mu \mathrm{M})$ treatment, followed by further incubation with DMSO or UA $(20 \mu \mathrm{M})$ for $24 \mathrm{~h}$. The values represent the mean \pm SD from two independent experiments performed in triplicate; ${ }^{*} p<0.05$ and ${ }^{* *} p<0.01$. (E) Intracellular total cholesterol levels were measured in UA or simvastatin-treated SK-HEP-1, Huh7 and Hep3B cells. $\mathrm{UA}$ or simvastatin was treated for $48 \mathrm{~h}$ prior to measure intracellular total cholesterol. The values are represented as the mean \pm SD of three independent experiments performed in duplicate; ${ }^{*} p<0.05$.

\subsection{UA Attenuates Growth-Signaling Pathways in A Cholesterol-Dependent Manner}

Cholesterol, a component of lipid rafts, promotes cell growth signaling factors, such as PI3K/AKT and EGFR/MAPK, by mediating the trafficking of oncogenic growth factor receptors [24]. In addition, multiple types of metabolites and intermediates generated from mevalonate pathway and cholesterol biosynthesis are critical for the membrane anchoring of oncogenic signaling proteins such as RAS, PI3K, and AKT [53]. Therefore, this study investigated whether UA could attenuate growth-factor-mediated oncogenic growth signaling pathways. As expected, the starvation of fetal bovine serum (FBS), 
which contains various growth factors, sufficiently decreased the phosphorylation of AKT, MEK, and ERK1/2, thereby increasing the FBS supplementation dramatically in these signaling pathways (Figure 3A-C). Interestingly, UA was found to suppress the phosphorylation of AKT, MEK, and ERK1/2 by growth factors-enriched FBS in SK-HEP-1 (Figure 3A), Huh7 (Figure 3B), and Hep3B (Figure 3C) cells. As hyperactivation of oncogenic signaling caused by gain-of-function genetic mutations such as PI3K, RAS, MYC, and RAF, is a hallmark of cancer development and progression [54], these results suggest that the suppressive effect of UA on oncogenic growth signaling is a critical insight for the development of UA-based anti-cancer therapeutics. However, there remains a question as to how UA downregulates the growth-factor-induced oncogenic signaling pathways. To understand this question, this study investigated whether cholesterol is responsible for the suppression of oncogenic growth signaling caused by UA. Figure 3D shows that the supplementation with water-soluble cholesterol was sufficient to reverse UA-induced attenuation of growth signaling in SK-HEP-1 and Hep3B cells. These results indicate that the cholesterol-lowering effect of UA is a possible regulatory mechanism by which UA suppresses oncogenic growth signaling in HCC cells.

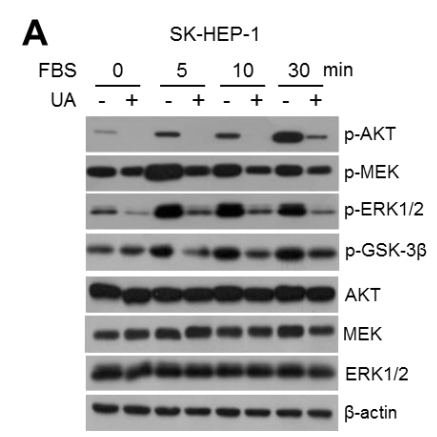

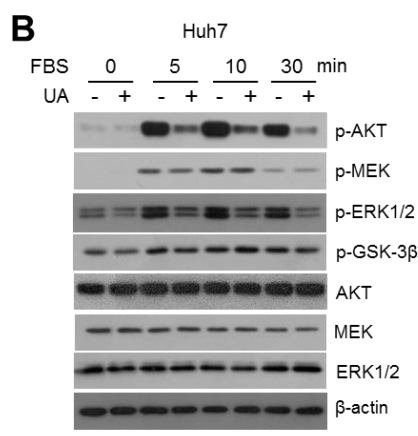

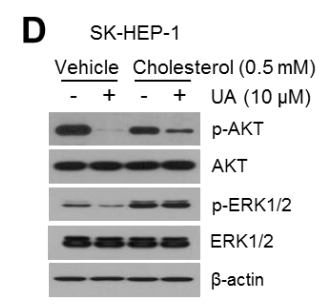

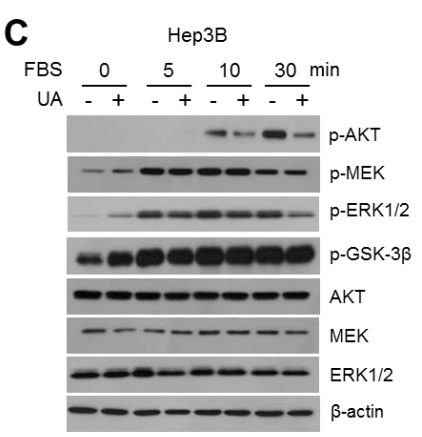

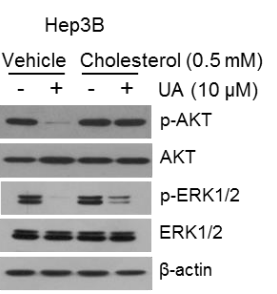

Figure 3. Ursolic acid (UA) increases the expression of cholesterol biosynthesis-related genes and enzymes in hepatocellular carcinoma (HCC) cells. (A) SK-HEP-1 cells were incubated, upon fetal bovine serum (FBS) starvation, for $24 \mathrm{~h}$ prior to UA treatment. After serum starvation, the cells incubated with DMSO or UA $(20 \mu \mathrm{M})$ in the absence or presence of $10 \% \mathrm{FBS}$, as indicated. The phosphorylation of growth signaling pathway-related proteins were measured by western blotting. (B) Suppressive effect of UA on growth signaling measured in Huh7. (C) Suppressive effect of UA on growth signaling measured in Hep3B. (D) SK-HEP-1 and Hep3B cells were incubated for $2 \mathrm{~h}$ with or without $0.5 \mathrm{mM}$ of water-soluble cholesterol prior to UA treatment, and cells were then further incubated with DMSO or UA $(20 \mu \mathrm{M})$ for $24 \mathrm{~h}$. Phosphorylation of AKT and ERK1/2 was measured using western blotting.

\subsection{UA Decreases Viability of Hepatocellular Carcinoma (HCC) Cells}

As UA dramatically suppresses the oncogenic growth signaling, the growth-suppressive effect of UA in HCC cells was further tested. Here, this study found that UA, at a concentration of 10-20 $\mu \mathrm{M}$, strongly inhibits cell viability in SK-HEP-1 (Figure 4A), Huh7 (Figure 4B), and Hep3B (Figure 4C) cells. Although the regulatory mechanism by which UA exerts anti-proliferative effects is not clear, large numbers of previous evidences have also shown that UA attenuates cell growth in multiple types of cancer cells, such as colorectal [11], breast [55], gastric [56], and melanoma [57]. Consistently, our results also show that UA was sufficient to decrease cell viability in lung (A549 and H1666), breast (MCF7 and MDA-MB-231), melanoma (A375 and A2058) and cervical (HeLa) cancer cells (Figure 4D). 
Thus, our findings, as well as those from other studies, support the potential efficacy of UA as a natural compound for the prevention and treatment of HCC. Previous reports have shown that $20 \mathrm{mg} / \mathrm{kg}$ of UA exerts anti-cancer effects in TC-1 (cervical cancer cells) and SW620 (colorectal cancer cells) transplanted mice models [58,59]. These backgrounds encourage the investigation of whether $20 \mathrm{mg} / \mathrm{kg}$ of UA effectively suppresses the growth of HCC and liver neoplasm by inhibiting mevalonate and cholesterol biosynthesis pathways in vivo models.
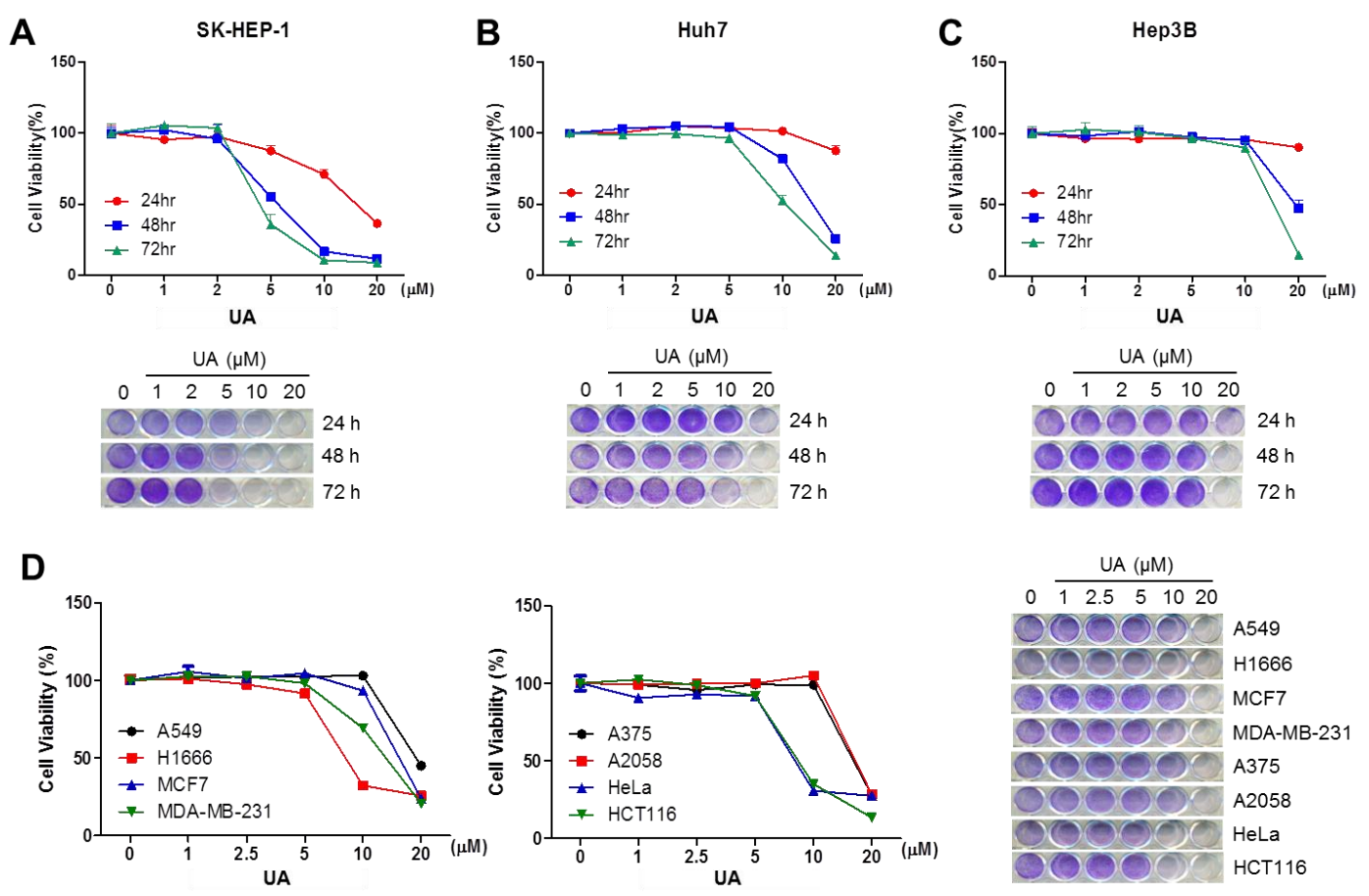

Figure 4. UA attenuates cell viability in HCC cells. (A) SK-HEP-1 cells were incubated with UA at different concentrations for 24, 48, and $73 \mathrm{~h}$, as indicated. (B) Attenuation of cell viability by UA treatment was measured in Huh7 cells. (C) Attenuation of cell viability by UA treatment was measured in Hep3B cells. (D) Attenuation of cell viability by UA treatment was measured in lung (A549 and H1666), breast (MCF7 and MDA-MB-231), melanoma (A375 and A2058), cervical (HeLa) and colon (HCT116) cancer cells. Cell viability was measured by crystal violet staining, and the values represent the mean from three independent experiments performed in duplicate.

\subsection{UA Promotes Cell Cycle Arrest and Apoptotic Death in HCC Cells}

The insufficient cholesterol biosynthesis and supplementation increases the incidence of cell cycle arrest and apoptotic cell death $[60,61]$. In addition, cholesterol-lowering drugs, such as simvastatin and lovastatin, have also been found to promote cell cycle arrest and apoptosis in various cancer cells [62-65]. Here, whether UA acts similarly to cholesterol-lowering drugs to promote cell cycle arrest and apoptotic cell death in HCC cells was investigated. Figure 5A,B shows that UA promotes cell cycle arrest at G0/G1 checkpoint in a dose-dependent manner. Consistent with this, approximately $20 \%$ increase in apoptotic cells upon treatment with $20 \mu \mathrm{M}$ UA, was observed in SK-HEP-1 cells (Figure 5C). These results indicate that UA causes cell cycle arrest and apoptotic cell death in HCC cells. 
A

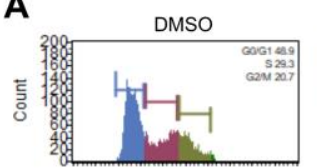

UA $10 \mu \mathrm{M}$

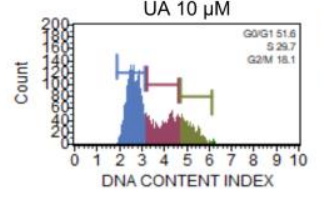

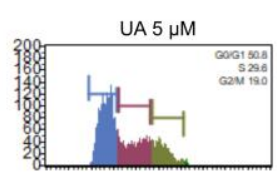

UA $20 \mu \mathrm{M}$

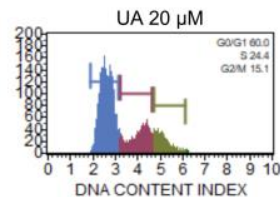

B

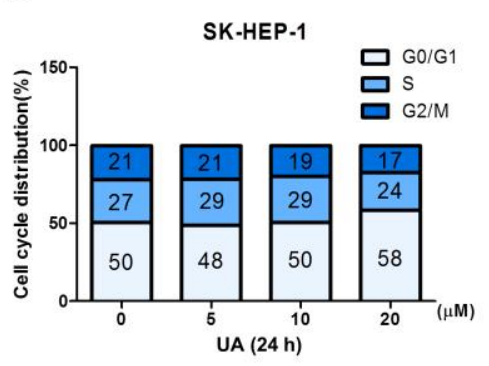

C

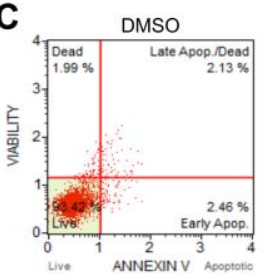

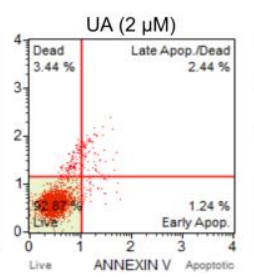
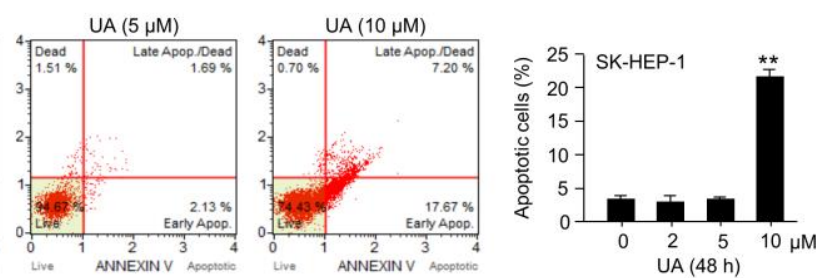

Figure 5. UA causes cell cycle arrest and apoptotic death in HCC cells. (A,B) SK-HEP-1 cells were incubated for $12 \mathrm{~h}$ with DMSO or UA at different concentrations, as indicated. Cell cycle was assessed using the Muse ${ }^{\mathrm{TM}}$ cell cycle assay kit. The values represent the mean from three independent experiments performed in duplicate. (C) SK-HEP-1 cells were incubated for $48 \mathrm{~h}$ with DMSO or UA at different concentrations, as indicated. Apoptotic cell death was measured using Muse ${ }^{\mathrm{TM}}$ Annexin V and Dead Cell kits. The values represent the mean \pm SD from three independent experiments performed in duplicate; ${ }^{* *} p<0.01$.

\subsection{Anti-Cancer Effect of UA in HCC Cells is Diminished by Cholesterol Supplementation}

To understand whether UA suppresses HCC cell growth through a cholesterol-lowering effect, the alteration in cell growth by UA was further measured in the absence or presence of cholesterol. Initially, a strong suppression of cell viability by UA upon FBS deprivation, was observed in SK-HEP-1, Hep3B, and Huh7 cells. This indicates that FBS, containing high levels of cholesterol in the form of lipoproteins may modulate the anti-cancer effects of UA (Figure 6A). Importantly, Figure 6B shows that UA attenuated the cell growth more effectively in the presence of lipoprotein-depleted FBS (DL-FBS) than in the culture medium containing normal FBS. As direct evidence of the fact that the cholesterol-lowering effect is a critical mechanism for the anti-cancer effect of UA, a decreased cell viability by UA was confirmed upon additional cholesterol supplementation. Consistent with Figure 6A,B, water-soluble cholesterol supplementation significantly reverses UA-induced attenuation of cell growth in HCC cells (Figure 6C). The evidence from previous studies indicates that UA inhibits cancer growth through its effects on oncogenic growth signaling and several cancer-promoting transcription factors, such as STAT3, NF-Kb, and HIF-1 $\alpha$ [5]. These results suggest that UA suppresses the oncogenic growth signaling through cholesterol-lowering effects, resulting in an attenuated growth of HCC cells. However, the critical regulatory mechanism by which UA attenuates cancer growth is poorly understood. Here, the authors speculate several possible regulatory mechanisms (Figure 6D). First, UA acts as an inhibitor of HMGCR similar to those of statins. Indeed, our results showed that UA decreased intracellular cholesterol levels, but paradoxically increased mevalonate and cholesterol biosynthesis-related genes expression, which are commonly observed in statins-treated cells. Second, UA could suppress FDPS-mediated production of FPP and GGPP, which activate oncogenic growth signaling and cellular transformation through farnesylation of oncogenic proteins including Ras [24]. In fact, it was found that oncogenic growth signaling such as RAS-MAPK and PI3K-AKT signaling pathways was diminished by UA treatment. Thus, these speculated regulatory mechanisms by which UA acts as an inhibitor against HMGCR and FDPS needed further in-depth investigations. 

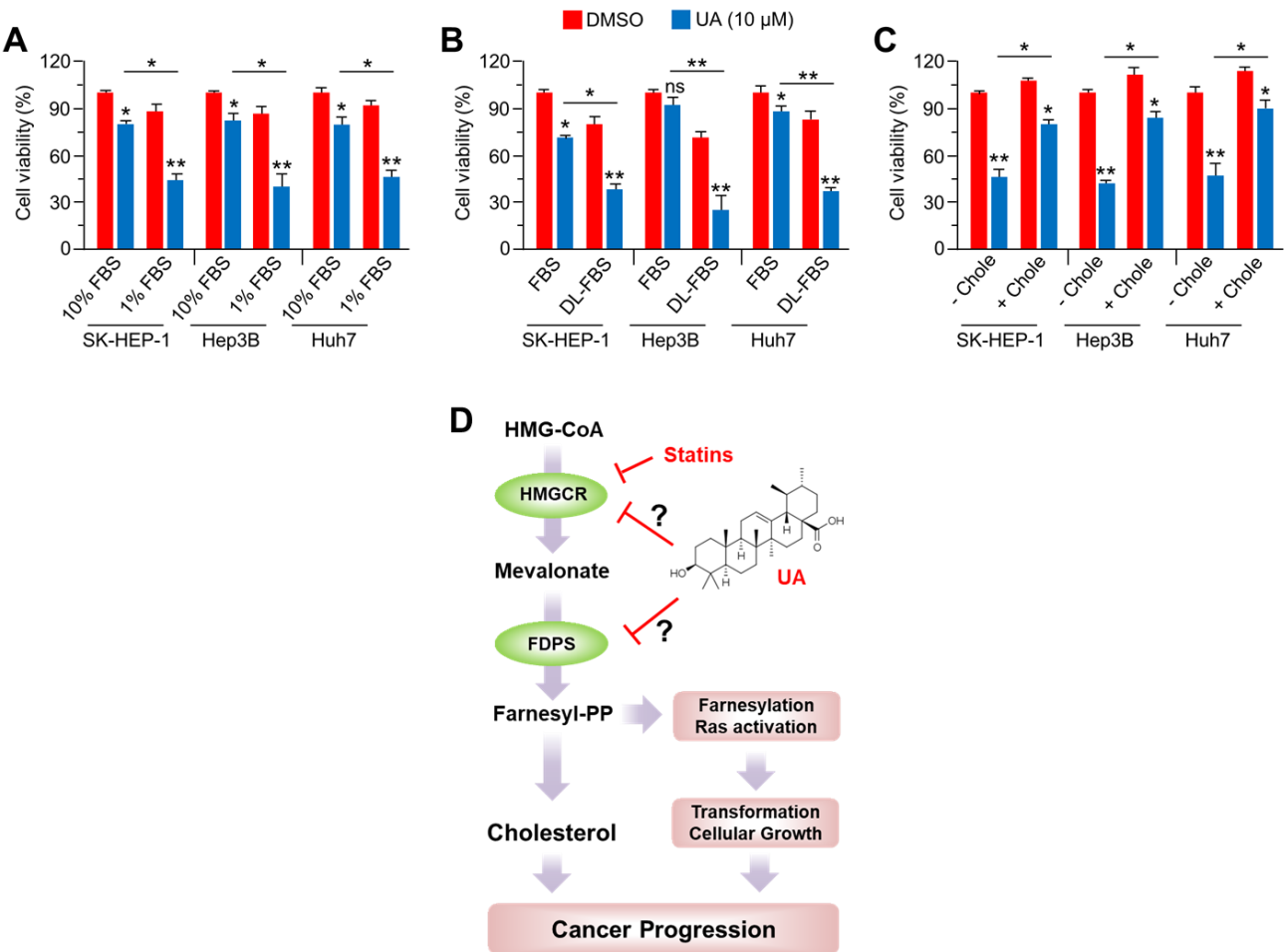

Figure 6. Cholesterol is required for the anti-cancer effect of UA. (A) Three cell lines (SK-HEP-1, Hep3B, and Huh7) were incubated with DMSO or UA $(20 \mu \mathrm{M})$ for $24 \mathrm{~h}$ with $10 \%$ FBS or $1 \%$ FBS. Cell viability was measured by crystal violet staining, and the values represent the mean \pm SD from three independent experiments performed in triplicate; ${ }^{*} p<0.05$ and ${ }^{* *} p<0.01$. (B) Cells were incubated with DMSO or UA $(20 \mu \mathrm{M})$ for $24 \mathrm{~h}$ with $10 \%$ normal FBS or lipoprotein-depleted FBS (DL-FBS). The values represented the mean \pm SD from three independent experiments performed in triplicate; ${ }^{*} p<0.05$ and ${ }^{* *} p<0.01$. (C) Cells were incubated with or without $0.5 \mathrm{mM}$ water-soluble cholesterol for $2 \mathrm{~h}$ prior to UA $(20 \mu \mathrm{M})$ treatment, followed by further incubation with DMSO or UA $(20 \mu \mathrm{M})$ for $24 \mathrm{~h}$ with $1 \%$ FBS. The values represent the mean \pm SD from two independent experiments performed in triplicate; ${ }^{*} p<0.05$ and ${ }^{* *} p<0.01$. (D) Speculated molecular mechanism of anti-cancer effects of UA.

\section{Materials and Methods}

\subsection{Reagents and Antibodies}

A library of 502 natural compounds was obtained from Enzo Biochem (Farmingdale, NY, USA). Ursolic acid (U6753), water-soluble cholesterol (C4951) and simvastatin (S6196) were purchased from Sigma Aldrich (St. Louis, MO, USA) and Santa Cruz Biotechnology (Dallas, TX, USA). The antibodies recognizing HMGCS1 (CST-36877), HMGCR (ab174830), FDPS (ab189874), SREBP2 (ab30682), AKT (CST-4691), phospho-AKT (CST-4060), MEK (CST-9122), phospho-MEK (CST-9154), ERK1/2 (sc-94), phospho-ERK1/2 (CST-4370), phospho-GSK3 $\beta$ (CST-5558) and $\beta$-actin (sc-47778) were purchased from Cell Signaling Technology (Danvers, MA, USA), Santa Cruz Biotechnology, and Abcam (Cambridge, MA, USA).

\subsection{Cell Culture and Cell Viability Assay}

Hepatocellular carcinoma (Hep3B, SK-HEP-1 and Huh7), lung adenocarcinoma (A549 and NCI-H1666), malignant melanoma (A375 and A2058), breast cancer (MCF7 and MDA-MB-231) and cervical cancer (HeLa) cells were obtained from the Korean Cell Line Bank (Seoul, Korea) and American Type Culture Collection (Manassas, VA, USA) and cultured in Dulbecco's modified Eagle's medium (DMEM) and Minimum Essential Medium Eagle alpha medium (MEM- $\alpha$ ) supplemented with 10\% fetal bovine serum (FBS) and antibiotics. Lipoprotein depleted FBS $(\mathrm{d} \leq 1.25 \mathrm{~g} / \mathrm{mL})$, which produced 
and qualified by ultracentrifugation and agarose gel electrophoresis, respectively, (DL-FBS, Kalen Biomedical, Germantown, MD, USA) was used for cholesterol deprivation. Cell viability was measured as previously described [1]. To measure cell viability, the Hep3B, SK-HEP-1 and Huh7 cells were seeded into 24-well tissue culture dishes and incubated for 24,48 , and $72 \mathrm{~h}$ with or without ursolic acid (UA) upon 10\% FBS, 1\% FBS, lipoprotein depletion and/or supplementation of water-soluble cholesterol. The cultured cells were washed and fixed with phosphate-buffered saline (PBS) and $4 \%$ paraformaldehyde. The fixed cells were incubated with crystal violet solution for $20 \mathrm{~min}$ at room temperature, and the stained cells were solubilized in $1 \%$ SDS solution. The optical density was measured at $570 \mathrm{~nm}$ by using an absorbance reader (BioTek, Winooski, VT, USA) (OD570).

\subsection{Western Blotting}

For western blotting, total protein samples were extracted by using a protein extraction buffer $(1 \%$ IGEPAL, $150 \mathrm{mM} \mathrm{NaCl}, 50 \mathrm{mM}$ Tris- $\mathrm{HCl}$ (pH 7.9), $10 \mathrm{mM} \mathrm{NaF}, 0.1 \mathrm{mM}$ EDTA, and a protease inhibitor cocktail) as previously described [1]. The total proteins were separated followed by molecular weight by using sodium dodecyl sulfate (SDS)-polyacrylamide gel electrophoresis (PAGE). The separated proteins were then transferred onto PVDF membranes (Millipore, Burlington, MA, USA), and the transferred membranes reacted with primary antibodies $(1: 1,000-1: 5,000)$ and horseradish peroxidase (HRP)-conjugated secondary antibodies $(1: 10,000)$ at $4{ }^{\circ} \mathrm{C}$ and room temperature for $12 \mathrm{~h}$ and $1 \mathrm{~h}$, respectively. The Enhanced Chemiluminescence (ECL) Prime kit (GE Healthcare, Pittsburgh, PA, USA) was used for visualization of the differences of protein expression.

\subsection{Quantitative Real-Time PCR}

The mRNA encoding cholesterol biosynthesis-related enzymes expression was measured by quantitative real-time polymerase chain reaction (PCR) performed as previously described [1]. Total RNA was extracted by using TRIzol (Invitrogen, Carlsbad, CA, USA). The cDNA was synthesized by using a high-capacity cDNA reverse transcription kit (Applied Biosystems, Waltham, MA, USA). Quantitative PCR was performed by using SYBR Green PCR Master Mix (Applied Biosystems, Waltham, MA, USA). Human 36B4 (rplp0, acidic ribosomal phosphoprotein P0) gene was used for housekeeping control. The primer sequences used in the experiment are shown in Table 1.

Table 1. Primer sequences for quantitative real time-PCR.

\begin{tabular}{ccc}
\hline Gene & Forward Primer & Reverse Primer \\
\hline HMGCS1 & TGGCAGGGAGTCTTGGTA & TCCCACTCCAAATGATGACA \\
HMGCR & GATGGGAGGCCACAAAGAG & TTCGGTGGCCTCTAGTGAGA \\
MVD & TTAACTGGTCCTGGTGCAGA & AACATCGCGGTCATCAAGTA \\
FDPS & TCCATGATGTCATCTGCCAC & AGCCAAGGAAACAGGATG \\
SREBP1a & GCACCCACTCCATTGAAGAT & GGCACTGACTCTTCCTTGATAC \\
SREBP1c & ACAGTGACTTCCCTGGCCTAT & GCATGGACGGGTACATCTTCA \\
SREBP2 & AACGGTCATTCACCCAGGTC & GGCTGAAGAATAGGAGTTGCC \\
LDL-R & AACTGCCATTGTCGTCTTTA & ACATACCCATCAACGACAAG \\
36B4 & CATGTTGCTGGCCAATAAGG & TGGTGATACCTAAAGCCTGGAA \\
\hline
\end{tabular}

\subsection{Cell Cycle Analysis}

The cell cycle analysis was performed as previously described [1]. The cultured cells in the absence or presence of UA were harvested and fixed using cold PBS and 70\% ethanol, and the cells were then incubated for $3 \mathrm{~h}$ at $-20^{\circ} \mathrm{C}$. The fixed cells were incubated with Muse ${ }^{\mathrm{TM}}$ cell cycle assay kit reagent $(200 \mu \mathrm{L})$ for $30 \mathrm{~min}$ at room temperature. The cell populations were analyzed by using a Mini Flow Cytometry Muse ${ }^{\mathrm{TM}}$ Cell Analyzer (Millipore, Burlington, MA, USA). 


\subsection{Apoptosis Assays}

Annexin-V staining to measure apoptotic cell death was performed as previously described [1]. The culture cells $\left(1 \times 10^{5}\right.$ cells/well $)$ in the absence or presence of UA were collected into fresh tubes, and the cells were then washed with cold PBS and centrifuged at $2000 \mathrm{rpm}$ for $2 \mathrm{~min}$ at room temperature. The cell pellets were mixed and reacted with $100 \mu \mathrm{L}$ of Muse ${ }^{\mathrm{TM}}$ Annexin V and Dead Cell kit reagents (Millipore, Burlington, MA, USA) for $20 \mathrm{~min}$. The apoptotic cells population was measured by using Mini Flow Cytometry Muse ${ }^{\mathrm{TM}}$ Cell Analyzer (Millipore, Burlington, MA, USA).

\subsection{Luciferase Assay}

Luciferase assay was performed as previously described [1]. HEK293T cells transiently transfected with steroid responsive element (SRE)-wild type (WT) or -mutant (Mut) containing luciferase vector (pSynSRE-T-Luc and pSynSRE-Mut-T-Luc) by using Lipofectamine 2000 (Invitrogen, Carlsbad, CA, USA). pSynSRE-T-Luciferase vectors (Addgene plasmid \#60444 and \#60490) were gifts from Timothy Osborne [66]. To screen the SREBP2 activating natural compounds, transfected HEK293T cells were incubated with a library of 502 natural compounds ( $20 \mu \mathrm{M}$ of each compounds) for $24 \mathrm{~h}$. Luciferase activities were analyzed by using a Synergy 2 Luminometer (BioTek, Winooski, VT, USA) and $\beta$-gal assay was used for normalization.

\subsection{Measurement of Intracellular Cholesterol}

Intracellular total cholesterol levels were measured by using the Amplex Red cholesterol assay kit (Invitrogen, Carlsbad, CA, USA) in accordance with the manufacturer's protocols as previously described [1]. Briefly, UA or simvastatin-treated SK-HEP-1, Huh7 and Hep3B cells were harvested and kept at $-70^{\circ} \mathrm{C}$. The cells were lysed with reaction buffer and sonication to disrupt the cellular membrane. The cell lysates $(50 \mu \mathrm{L})$ were mixed and reacted for $30 \mathrm{~min}$ at $37^{\circ} \mathrm{C}$ with horseradish peroxidase (HRP), cholesterol oxidase and cholesterol esterase containing Amplex Red reagent, and then the fluorescence intensity was measured by using a fluorescence microplate reader (BioTek, Winooski, VT, USA) at ex/em $=530 / 590$.

\subsection{Statistical Analysis}

The data are represented as the mean \pm standard deviation (SD). All statistical analyses were performed using two-tailed Student's $t$-test and one-way ANOVA with Tukey post hoc test. A $p$ value of $<0.05$ was considered statistically significant.

\section{Conclusions}

Despite the accumulating evidence that UA exerts an anti-cancer effect in multiple types of cancer, the precise underlying molecular mechanism is not clear. The major finding of this study is that UA exerts its anti-cancer effect through a cholesterol-lowering effect in hepatocellular carcinoma (HCC) cells. Taken together, these results indicate that UA, as a promising cholesterol-lowering drug, may be useful for the development of drugs and/or functional foods for the prevention and treatment of HCC as well as dysregulated cholesterol metabolism-related chronic diseases.

Author Contributions: Conceptualization, J.-H.L.; formal analysis, G.-H.K., J.H.K., H.M.K. and J.-H.L.; methodology, G.-H.K., S.L., S.-Y.K., H.M.K. and H.K.; supervision, J.-H.L.; writing-original draft, J.-H.L.; writing-review and editing, J.H.K. and J.-H.L.

Funding: This work was supported by the National Research Foundation of Korea (NRF) grant funded by the Korea government (2016R1A5A2012284) and Basic Science Research Program through the National Research Foundation of Korea (NRF) funded by the Ministry of Education (2018R1D1A1A02085330).

Conflicts of Interest: The authors declare no conflict of interest. 


\section{References}

1. Kim, Y.S.; Lee, Y.M.; Oh, T.I.; Shin, D.H.; Kim, G.H.; Kan, S.Y.; Kang, H.; Kim, J.H.; Kim, B.M.; Yim, W.J.; et al. Emodin Sensitizes Hepatocellular Carcinoma Cells to the Anti-Cancer Effect of Sorafenib through Suppression of Cholesterol Metabolism. Int. J. Mol. Sci. 2018, 19, 3127. [CrossRef] [PubMed]

2. Forner, A.; Llovet, J.M.; Bruix, J. Hepatocellular carcinoma. Lancet 2012, 379, 1245-1255. [CrossRef]

3. Greten, T.F.; Korangy, F.; Manns, M.P.; Malek, N.P. Molecular therapy for the treatment of hepatocellular carcinoma. Br. J. Cancer 2009, 100, 9-23. [CrossRef] [PubMed]

4. Woźniak, Ł.; Skapska, S.; Marszałek, K. Ursolic Acid-A Pentacyclic Triterpenoid with a Wide Spectrum of Pharmacological Activities. Molecules 2015, 20, 20614-20641. [CrossRef] [PubMed]

5. Yadav, V.R.; Prasad, S.; Sung, B.; Kannappan, R.; Aggarwal, B.B. Targeting Inflammatory Pathways by Triterpenoids for Prevention and Treatment of Cancer. Toxins 2010, 2, 2428-2466. [CrossRef]

6. Ruan, J.S.; Zhou, H.; Yang, L.; Wang, L.; Jiang, Z.S.; Sun, H.; Wang, S.M. Ursolic Acid Attenuates TGF- $\beta 1$-Induced Epithelial-Mesenchymal Transition in NSCLC by Targeting Integrin $\alpha$ V $\beta 5 / M M P s$ Signaling. Oncol. Res. Featur. Preclin. Clin. Cancer Ther. 2019, 27, 593-600. [CrossRef]

7. Song, B.; Zhang, Q.; Yu, M.; Qi, X.; Wang, G.; Xiao, L. Ursolic acid sensitizes radioresistant NSCLC cells expressing HIF-1 $\alpha$ through reducing endogenous GSH and inhibiting HIF-1 $\alpha$. Oncol. Lett. 2017, 13, 754-762. [CrossRef]

8. Zong, L.; Cheng, G.; Liu, S.; Pi, Z.; Liu, Z.; Song, F. Reversal of multidrug resistance in breast cancer cells by a combination of ursolic acid with doxorubicin. J. Pharm. Biomed. Anal. 2019, 165, 268-275. [CrossRef]

9. Manouchehri, J.M.; Kalafatis, M. Ursolic Acid Promotes the Sensitization of rhTRAIL-resistant Triple-negative Breast Cancer. Anticancer Res. 2018, 38, 6789-6795. [CrossRef]

10. Meng, Y.; Lin, Z.M.; Ge, N.; Zhang, D.L.; Huang, J.; Kong, F. Ursolic Acid Induces Apoptosis of Prostate Cancer Cells via the PI3K/Akt/mTOR Pathway. Am. J. Chin. Med. 2015, 43, 1471-1486. [CrossRef]

11. Kim, K.; Shin, E.A.; Jung, J.H.; Park, J.E.; Kim, D.S.; Shim, B.S.; Kim, S.H. Ursolic Acid Induces Apoptosis in Colorectal Cancer Cells Partially via Upregulation of MicroRNA-4500 and Inhibition of JAK2/STAT3 Phosphorylation. Int. J. Mol. Sci. 2018, 20, 114. [CrossRef] [PubMed]

12. Prasad, S.; Yadav, V.R.; Sung, B.; Reuter, S.; Kannappan, R.; Deorukhkar, A.; Diagaradjane, P.; Wei, C.; Baladandayuthapani, V.; Krishnan, S.; et al. Ursolic acid inhibits growth and metastasis of human colorectal cancer in an orthotopic nude mouse model by targeting multiple cell signaling pathways: Chemosensitization with capecitabine. Clin. Cancer Res. 2012, 18, 4942-4953. [CrossRef] [PubMed]

13. Liu, T.; Ma, H.; Shi, W.; Duan, J.; Wang, Y.; Zhang, C.; Li, C.; Lin, J.; Lv, J. Inhibition of STAT3 signaling pathway by ursolic acid suppresses growth of hepatocellular carcinoma. Int. J. Oncol. 2017, 51, 555-562. [CrossRef] [PubMed]

14. Yie, Y.; Zhao, S.; Tang, Q.; Zheng, F.; Wu, J.; Yang, L. Ursolic acid inhibited growth of hepatocellular carcinoma HepG2 cells through AMPK $\alpha$-mediated reduction of DNA methyltransferase 1. Mol. Cell. Biochem. 2015, 402, 63-74. [CrossRef] [PubMed]

15. Xu, X.; Zhu, G.Q.; Zhang, K.; Zhou, Y.C.; Li, X.L.; Xu, W.; Zhang, H.; Shao, Y.; Zhang, Z.Y.; Sun, W.H. Cyclooxygenase-2 mediated synergistic effect of ursolic acid in combination with paclitaxel against human gastric carcinoma. Oncotarget 2017, 8, 92770-92777. [CrossRef] [PubMed]

16. Junco, J.J.; Cho, J.; Mancha, A.; Malik, G.; Wei, S.J.; Kim, D.J.; Liang, H.; DiGiovanni, J.; Slaga, T.J. Role of AMPK and PPAR $\alpha$ in the anti-skin cancer effects of ursolic acid. Mol. Carcinog. 2018, 57, 1698-1706. [CrossRef] [PubMed]

17. Huang, M.T.; Ho, C.T.; Wang, Z.Y.; Ferraro, T.; Lou, Y.R.; Stauber, K.; Ma, W.; Georgiadis, C.; Laskin, J.D.; Conney, A.H. Inhibition of skin tumorigenesis by rosemary and its constituents carnosol and ursolic acid. Cancer Res. 1994, 54, 701-708.

18. Rajagopal, C.; Lankadasari, M.B.; Aranjani, J.M.; Harikumar, K. Targeting oncogenic transcription factors by polyphenols: A novel approach for cancer therapy. Pharmacol. Res. 2018, 130, 273-291. [CrossRef]

19. Shanmugam, M.K.; Manu, K.A.; Ong, T.H.; Ramachandran, L.; Surana, R.; Bist, P.; Lim, L.H.; Kumar, A.P.; Sethi, G.; Hui, K.M. Inhibition of CXCR4/CXCL12 signaling axis by ursolic acid leads to suppression of metastasis in transgenic adenocarcinoma of mouse prostate model. Int. J. Cancer 2011, 129, 1552-1563. [CrossRef]

20. Silvente-Poirot, S.; Poirot, M. Cholesterol and Cancer, in the Balance. Science 2014, 343, 1445-1446. [CrossRef] 
21. Murai, T. Cholesterol lowering: Role in cancer prevention and treatment. Boil. Chem. 2015, 396, 1-11. [CrossRef] [PubMed]

22. Zhuang, L.; Lin, J.; Lu, M.L.; Solomon, K.R.; Freeman, M.R. Cholesterol-rich lipid rafts mediate akt-regulated survival in prostate cancer cells. Cancer Res. 2002, 62, 2227-2231. [PubMed]

23. Zhuang, L.; Kim, J.; Adam, R.M.; Solomon, K.R.; Freeman, M.R. Cholesterol targeting alters lipid raft composition and cell survival in prostate cancer cells and xenografts. J. Clin. Investig. 2005, 115, 959-968. [CrossRef] [PubMed]

24. Mullen, P.J.; Yu, R.; Longo, J.; Archer, M.C.; Penn, L.Z. The interplay between cell signalling and the mevalonate pathway in cancer. Nat. Rev. Cancer 2016, 16, 718-731. [CrossRef] [PubMed]

25. Desvergne, B.; Michalik, L.; Wahli, W. Transcriptional Regulation of Metabolism. Physiol. Rev. 2006, 86, 465-514. [CrossRef] [PubMed]

26. Qi, X.F.; Zheng, L.; Lee, K.J.; Kim, D.H.; Kim, C.S.; Cai, D.Q.; Wu, Z.; Qin, J.W.; Yu, Y.H.; Kim, S.K. HMG-CoA reductase inhibitors induce apoptosis of lymphoma cells by promoting ROS generation and regulating Akt, Erk and p38 signals via suppression of mevalonate pathway. Cell Death Dis. 2013, 4, e518. [CrossRef] [PubMed]

27. Bai, F.; Yu, Z.; Gao, X.; Gong, J.; Fan, L.; Liu, F. Simvastatin induces breast cancer cell death through oxidative stress up-regulating miR-140-5p. Aging 2019, 11, 3198-3219. [CrossRef] [PubMed]

28. Kotamraju, S.; Willams, C.L.; Kalyanaraman, B. Statin-Induced Breast Cancer Cell Death: Role of Inducible Nitric Oxide and Arginase-Dependent Pathways. Cancer Res. 2007, 67, 7386-7394. [CrossRef]

29. Jiang, S.; Wang, X.; Song, D.; Liu, X.; Gu, Y.; Xu, Z.; Wang, X.; Zhang, X.; Ye, Q.; Tong, Z.; et al. Cholesterol Induces Epithelial-to-Mesenchymal Transition of Prostate Cancer Cells by Suppressing Degradation of EGFR through APMAP. Cancer Res. 2019, 79, 3063-3075. [CrossRef]

30. Wang, B.; Rong, X.; Palladino, E.N.; Wang, J.; Fogelman, A.M.; Martin, M.G.; Alrefai, W.A.; Ford, D.A.; Tontonoz, P. Phospholipid Remodeling and Cholesterol Availability Regulate Intestinal Stemness and Tumorigenesis. Cell Stem Cell 2018, 22, 206-220. [CrossRef]

31. Ehmsen, S.; Pedersen, M.H.; Wang, G.; Terp, M.G.; Arslanagic, A.; Hood, B.L.; Conrads, T.P.; Leth-Larsen, R.; Ditzel, H.J. Increased Cholesterol Biosynthesis Is a Key Characteristic of Breast Cancer Stem Cells Influencing Patient Outcome. Cell Rep. 2019, 27, 3927-3938. [CrossRef] [PubMed]

32. Mansourian, P.G.; Yoneda, M.; Rao, M.K.; Martinez, F.J.; Thomas, E.; Schiff, E.R. Effects of Statins on the Risk of Hepatocellular Carcinoma. Gastroenterol. Hepatol. 2014, 10, 417-426.

33. Manthravadi, S.; Shrestha, A.; Madhusudhana, S. Impact of statin use on cancer recurrence and mortality in breast cancer: A systematic review and meta-analysis. Int. J. Cancer 2016, 139, 1281-1288. [CrossRef] [PubMed]

34. McGlynn, K.A.; Divine, G.W.; Sahasrabuddhe, V.V.; Engel, L.S.; Vanslooten, A.; Wells, K.; Yood, M.U.; Alford, S.H. Statin use and risk of hepatocellular carcinoma in a U.S. population. Cancer Epidemiol. 2014, 38, 523-527. [CrossRef] [PubMed]

35. Nayan, M.; Punjani, N.; Juurlink, D.N.; Finelli, A.; Austin, P.C.; Kulkarni, G.S.; Uleryk, E.; Hamilton, R.J. Statin use and kidney cancer survival outcomes: A systematic review and meta-analysis. Cancer Treat. Rev. 2017, 52, 105-116. [CrossRef]

36. Cardwell, C.R.; Mc Menamin, Ú.; Hughes, C.M.; Murray, L.J. Statin Use and Survival from Lung Cancer: A Population-Based Cohort Study. Cancer Epidemiol. Biomark. Prev. 2015, 24, 833-841. [CrossRef] [PubMed]

37. Alfaqih, M.A.; Allott, E.H.; Hamilton, R.J.; Freeman, M.R.; Freedland, S.J. The current evidence on statin use and prostate cancer prevention: Are we there yet? Nat. Rev. Urol. 2017, 14, 107-119. [CrossRef]

38. Gray, R.T.; Coleman, H.G.; Hughes, C.; Murray, L.J.; Cardwell, C.R. Statin use and survival in colorectal cancer: Results from a population-based cohort study and an updated systematic review and meta-analysis. Cancer Epidemiol. 2016, 45, 71-81. [CrossRef]

39. Huang, W.Y.; Li, C.H.; Lin, C.L.; Liang, J.A. Long-term statin use in patients with lung cancer and dyslipidemia reduces the risk of death. Oncotarget 2016, 7, 42208-42215. [CrossRef]

40. Nielsen, S.F.; Nordestgaard, B.G.; Bojesen, S.E. Statin Use and Reduced Cancer-Related Mortality. N. Engl. J. Med. 2012, 367, 1792-1802. [CrossRef]

41. Cicero, A.F.G.; Colletti, A. An update on the safety of nutraceuticals and effects on lipid parameters. Expert Opin. Drug Saf. 2018, 17, 303-313. [CrossRef] [PubMed] 
42. Mascaró, C.; Ortiz, J.A.; Ramos, M.M.; Haro, D.; Hegardt, F.G. Sterol Regulatory Element Binding Protein-Mediated Effect of Fluvastatin on Cytosolic 3-Hydroxy-3-methylglutaryl-Coenzyme A Synthase Transcription. Arch. Biochem. Biophys. 2000, 374, 286-292. [CrossRef] [PubMed]

43. Born, E.; Murthy, S.; Mathur, S.N.; Field, F.J. Regulation of Sterol Regulatory Element-binding Proteins in Hamster Intestine by Changes in Cholesterol Flux. J. Boil. Chem. 2001, 276, 17576-17583.

44. Yu, S.G.; Zhang, C.J.; Xu, X.E.; Sun, J.H.; Zhang, L.; Yu, P.F. Ursolic acid derivative ameliorates streptozotocininduced diabestic bone deleterious effects in mice. Int. J. Clin. Exp. Pathol. 2015, 8, 3681-3690. [PubMed]

45. Sundaresan, A.; Radhiga, T.; Pugalendi, K.V. Effect of ursolic acid and Rosiglitazone combination on hepatic lipid accumulation in high fat diet-fed C57BL/6J mice. Eur. J. Pharmacol. 2014, 741, 297-303. [CrossRef] [PubMed]

46. Bakhtiari, N.; Hosseinkhani, S.; Soleimani, M.; Hemmati, R.; Noori-Zadeh, A.; Javan, M.; Tashakor, A. Short-term ursolic acid promotes skeletal muscle rejuvenation through enhancing of SIRT1 expression and satellite cells proliferation. Biomed. Pharmacother. 2016, 78, 185-196. [CrossRef] [PubMed]

47. Kazmi, I.; Afzal, M.; Rahman, S.; Iqbal, M.; Imam, F.; Anwar, F. Antiobesity potential of ursolic acid stearoyl glucoside by inhibiting pancreatic lipase. Eur. J. Pharmacol. 2013, 709, 28-36. [CrossRef]

48. Zhang, Y.; Song, C.; Li, H.; Hou, J.; Li, D. Ursolic acid prevents augmented peripheral inflammation and inflammatory hyperalgesia in high-fat diet-induced obese rats by restoring downregulated spinal PPAR $\alpha$. Mol. Med. Rep. 2016, 13, 5309-5316. [CrossRef]

49. Li, S.; Meng, F.; Liao, X.; Wang, Y.; Sun, Z.; Guo, F.; Li, X.; Meng, M.; Li, Y.; Sun, C. Therapeutic Role of Ursolic Acid on Ameliorating Hepatic Steatosis and Improving Metabolic Disorders in High-Fat Diet-Induced Non-Alcoholic Fatty Liver Disease Rats. PLoS ONE 2014, 9, e86724. [CrossRef]

50. Ma, J.Q.; Ding, J.; Zhang, L.; Liu, C.M. Protective effects of ursolic acid in an experimental model of liver fibrosis through Nrf2/ARE pathway. Clin. Res. Hepatol. Gastroenterol. 2015, 39, 188-197. [CrossRef]

51. Goldstein, J.L.; DeBose-Boyd, R.A.; Brown, M.S. Protein Sensors for Membrane Sterols. Cell 2006, 124, 35-46. [CrossRef] [PubMed]

52. Jiang, S.Y.; Li, H.; Tang, J.J.; Wang, J.; Luo, J.; Liu, B.; Wang, J.K.; Shi, X.J.; Cui, H.W.; Tang, J.; et al. Discovery of a potent HMG-CoA reductase degrader that eliminates statin-induced reductase accumulation and lowers cholesterol. Nat. Commun. 2018, 9, 5138. [CrossRef] [PubMed]

53. Gabitova, L.; Gorin, A.; Astsaturov, I. Molecular pathways: Sterols and receptor signaling in cancer. Clin. Cancer Res. 2014, 20, 28-34. [CrossRef] [PubMed]

54. Hanahan, D.; Weinberg, R.A. Hallmarks of Cancer: The Next Generation. Cell 2011, 144, 646-674. [CrossRef] [PubMed]

55. Xiang, F.; Fan, Y.; Ni, Z.; Liu, Q.; Zhu, Z.; Chen, Z.; Hao, W.; Yue, H.; Wu, R.; Kang, X. Ursolic Acid Reverses the Chemoresistance of Breast Cancer Cells to Paclitaxel by Targeting MiRNA-149-5p/MyD88. Front. Oncol. 2019, 9, 501. [CrossRef]

56. Li, J.; Dai, C.; Shen, L. Ursolic Acid Inhibits Epithelial-Mesenchymal Transition through the Axl/NF-kB Pathway in Gastric Cancer Cells. Evid. Based Complement. Altern. Med. 2019, 2019, 2474805. [CrossRef] [PubMed]

57. Tremmel, L.; Rho, O.; Slaga, T.J.; DiGiovanni, J. Inhibition of skin tumor promotion by TPA using a combination of topically applied ursolic acid and curcumin. Mol. Carcinog. 2019, 58, 185-195. [CrossRef]

58. Leng, S.; Hao, Y.; Du, D.; Xie, S.; Hong, L.; Gu, H.; Zhu, X.; Zhang, J.; Fan, D.; Kung, H.F. Ursolic acid promotes cancer cell death by inducing Atg5-dependent autophagy. Int. J. Cancer 2013, 133, 2781-2790. [CrossRef]

59. Shan, J.; Xuan, Y.; Zhang, Q.; Zhu, C.; Liu, Z.; Zhang, S. Ursolic acid synergistically enhances the therapeutic effects of oxaliplatin in colorectal cancer. Protein Cell 2016, 7, 571-585. [CrossRef]

60. Chen, H.W.; Kandutsch, A.A.; Waymouth, C. Inhibition of cell growth by oxygenated derivatives of cholesterol. Nature 1974, 251, 419-421. [CrossRef]

61. Brown, M.S.; Goldstein, J.L. Suppression of 3-hydroxy-3-methylglutaryl coenzyme A reductase activity and inhibition of growth of human fibroblasts by 7-ketocholesterol. J. Boil. Chem. 1974, 249, 7306-7314.

62. Peng, Y.; He, G.; Tang, D.; Xiong, L.; Wen, Y.; Miao, X.; Hong, Z.; Yao, H.; Chen, C.; Yan, S.; et al. Lovastatin Inhibits Cancer Stem Cells and Sensitizes to Chemo- and Photodynamic Therapy in Nasopharyngeal Carcinoma. J. Cancer 2017, 8, 1655-1664. [CrossRef]

63. Chen, M.J.; Cheng, A.C.; Lee, M.F.; Hsu, Y.C.; Chen, M.; Cheng, A.; Lee, M.; Hsu, Y. Simvastatin induces G1 arrest by up-regulating GSK3 $\beta$ and down-regulating CDK4/cyclin D1 and CDK2/cyclin E1 in human primary colorectal cancer cells. J. Cell. Physiol. 2018, 233, 4618-4625. [CrossRef] [PubMed] 
64. Fujiwara, D.; Tsubaki, M.; Tomonari, Y.; Koumoto, Y.I.; Sakaguchi, K.; Nishida, S.; Takeda, T. Statins induce apoptosis through inhibition of Ras signaling pathways and enhancement of Bim and p27 expression in human hematopoietic tumor cells. Tumor Boil. 2017, 39, 1010428317734947. [CrossRef] [PubMed]

65. Chen, M.C.; Tsai, Y.C.; Tseng, J.H.; Liou, J.J.; Horng, S.; Wen, H.C.; Fan, Y.C.; Zhong, W.B.; Hsu, S.P. Simvastatin Inhibits Cell Proliferation and Migration in Human Anaplastic Thyroid Cancer. Int. J. Mol. Sci. 2017, 18, 2690. [CrossRef] [PubMed]

66. Smith, J.R.; Osborne, T.F.; Brown, M.S.; Goldstein, J.L.; Gil, G. Multiple sterol regulatory elements in promoter for hamster 3-hydroxy-3-methylglutaryl-coenzyme A synthase. J. Boil. Chem. 1988, 263, 18480-18487.

(C) 2019 by the authors. Licensee MDPI, Basel, Switzerland. This article is an open access article distributed under the terms and conditions of the Creative Commons Attribution (CC BY) license (http://creativecommons.org/licenses/by/4.0/). 